Washington University School of Medicine Digital Commons@Becker

Open Access Publications

6-1-2019

\title{
Phase II trial of levocetirizine with capecitabine and bevacizumab to overcome the resistance of antiangiogenic therapies in refractory metastatic colorectal cancer
}

\author{
Manik Amin \\ Washington University School of Medicine in St. Louis \\ Monica Desai \\ Washington University School of Medicine in St. Louis \\ Kathryn Trinkaus \\ Washington University School of Medicine in St. Louis \\ Amberly Brown \\ Washington University School of Medicine in St. Louis \\ Andrea Wang-Gillam \\ Washington University School of Medicine in St. Louis
}

See next page for additional authors

Follow this and additional works at: https://digitalcommons.wustl.edu/open_access_pubs

Please let us know how this document benefits you.

\section{Recommended Citation}

Amin, Manik; Desai, Monica; Trinkaus, Kathryn; Brown, Amberly; Wang-Gillam, Andrea; Tan, Benjamin; Picus, Joel; Sorscher, Steven; Highkin, Maureen; Lears, Kim; and Lockhart, Albert C., "Phase II trial of levocetirizine with capecitabine and bevacizumab to overcome the resistance of antiangiogenic therapies in refractory metastatic colorectal cancer." Journal Gastrointestinal Oncology. 10, 3. 412 - 420. (2019). https://digitalcommons.wustl.edu/open_access_pubs/9921

This Open Access Publication is brought to you for free and open access by Digital Commons@Becker. It has been accepted for inclusion in Open Access Publications by an authorized administrator of Digital Commons@Becker. For more information, please contact vanam@wustl.edu. 


\section{Authors}

Manik Amin, Monica Desai, Kathryn Trinkaus, Amberly Brown, Andrea Wang-Gillam, Benjamin Tan, Joel Picus, Steven Sorscher, Maureen Highkin, Kim Lears, and Albert C. Lockhart 


\title{
Phase II trial of levocetirizine with capecitabine and bevacizumab to overcome the resistance of antiangiogenic therapies in refractory metastatic colorectal cancer
}

\author{
Manik Amin ${ }^{1}$, Monica Desai ${ }^{1}$, Kathryn Trinkaus ${ }^{2}$, Amberly Brown ${ }^{1}$, Andrea Wang-Gillam ${ }^{1}$, Benjamin Tan $^{1}$, \\ Joel Picus ${ }^{1}$, Steven Sorscher ${ }^{3}$, Maureen Highkin ${ }^{1}$, Kim Lears ${ }^{1}$, Albert C. Lockhart ${ }^{4}$ \\ ${ }^{1}$ Division of Medical Oncology, ${ }^{2}$ Biostatistics Shared Resource, Siteman Cancer Center, Washington University in Saint Louis, St. Louis, MO, USA; \\ ${ }^{3}$ Division of Hematology/Oncology, Wake Forest University, Winston-Salem, NC, USA; ${ }^{4}$ Division of Oncology, University of Miami Miller School \\ of Medicine, Miami, FL, USA \\ Contributions: (I) Conception and design: M Amin, M Desai, AC Lockhart; (II) Administrative support: AC Lockhart; (III) Provision of study \\ materials or patients: M Amin, M Desai, A Wang-Gillam, B Tan, J Picus, S Sorscher, AC Lockhart, A Brown; (IV) Collection and assembly of data: \\ M Amin, M Desai, K Trinkaus, A Brown, M Highkin, AC Lockhart; (V) Data analysis and interpretation: M Amin, AC Lockhart, K Trinkaus, A \\ Brown; (VI) Manuscript writing: All authors; (VII) Final approval of manuscript: All authors. \\ Correspondence to: Manik Amin, MD. Assistant Professor of Medicine, Division of Medical Oncology, Washington University School of Medicine, 660 \\ S. Euclid Ave Box 8056, St. Louis, MO 63110, USA. Email: manikamin@wustl.edu.
}

Background: Despite the clinical success of vascular endothelial growth factor (VEGF) blockade in metastatic colorectal cancers (mCRC), resistance to anti-angiogenic drugs invariably develops. IL- 8 and other cytokines have been implicated in development of resistance to anti-angiogenic therapy. Levocetirizine is a second generation $\mathrm{H} 1$ antihistamine with anti-inflammatory and IL-8 suppression properties. We conducted a phase II trial combining levocetirizine with capecitabine and bevacizumab to potentially overcome anti-angiogenic therapy resistance in patients with refractory mCRC.

Methods: This was a single-center open-label prospective trial in refractory mCRC patients. Treatment consisted of oral capecitabine $850 \mathrm{mg} / \mathrm{m}^{2}$ twice daily administered as 7 days on and 7 days off, intravenous (IV) bevacizumab $5 \mathrm{mg} / \mathrm{kg}$ every 14 days and oral levocetirizine $5 \mathrm{mg}$ daily. The primary end point was progression free survival (PFS) and secondary endpoints included objective response rate (ORR) and tolerability. An exploratory endpoint included correlation of PFS with cytokine levels. A sample size of 36 evaluable patients could identify a median PFS of 3.4 months at a 0.05 significance level. To examine cytokine changes related to levocetirizine treatment, patients were randomized to Arm A where levocetirizine was started 7 days after starting chemotherapy and to Arm B where levocetirizine was started 7 days prior to chemotherapy. Cytokine levels were measured at baseline and with each cycle of chemotherapy (up to three cycles).

Results: Forty-seven patients were enrolled in the trial to have 36 evaluable patients. Arm A enrolled 23 patients and Arm B enrolled 24 patients. Fifty percent of patients had progressive disease and $62 \%$ of patients had stable disease in each arm as best response. There was no demonstrable difference in PFS between the two arms (log-rank test $\mathrm{P}=0.83$ ). Median time to progression was 3.4 months in Arm A and 3.5 months in Arm B.

Conclusions: Median PFS in the trial was comparable to and appeared to be better than other regimens used in the refractory setting (e.g., median PFS of 1.9 months for regorafenib). Cytokine measurement with IL-8 levels did not show any correlation with progression free survival but patients with stable disease showed overall lower levels of IL-8 as compared to patients with progressive disease in the cytokine analysis.

Keywords: Levocetirizine; cytokine IL-8; anti-angiogenic resistance; metastatic colorectal cancer

Submitted Jan 09, 2019. Accepted for publication Feb 13, 2019.

doi: 10.21037/jgo.2019.02.01

View this article at: http://dx.doi.org/10.21037/jgo.2019.02.01 


\section{Introduction}

Colorectal cancer is currently third most common cancer and third leading cause of cancer-related death in the United States. It is estimated that 140,250 new cases of colorectal cancer will be diagnosed and 50,630 men and women will die of colorectal cancer in 2018 (1). Approximately $20 \%$ of patients will have distant disease at the time of presentation and of the remaining, 50-60\% will develop metastatic disease (2). Current treatment options for metastatic colorectal cancer include FOLFOX (5-FU/ leucovorin oxaliplatin), FOLFIRI (5-FU/leucovorin and irinotecan) chemotherapy with either angiogenesis inhibitor bevacizumab or an EGFR inhibitor such as cetuximab or panitumumab depending upon the RAS status (3-14). Combination of FOLFIRI plus aflibercept, FOLFIRI plus ramucirumab, regorafenib, trifluridine/tipiracil, nivolumab and pembrolizumab for MSI- $\mathrm{H}$ colorectal cancers are also FDA approved for second line or later in the treatment of metastatic colorectal cancer $(13,15-21)$. Addition of all the novel agents to the current chemotherapy, has increased median overall survival of patients with advanced colorectal cancer currently approaching three years (22). Despite significant advancements in the management of metastatic colorectal cancer, prognosis of metastatic colorectal cancer remains poor after progression of disease on first-line therapy (23).

Our study was initiated after FDA approval of regorafenib but prior to FDA approvals of trifluridine/ tipiracil, nivolumab and pembrolizumab for metastatic refractory colorectal cancer. Patients in our trial were considered refractory to the available chemotherapy options at that time and to preserve quality of life, we decided to use a regimen of oral capecitabine one week on and one week off and IV bevacizumab every 14 days which is considered as clinically active, is feasible to administer and also tolerable to the patients (24). A capecitabine dose of $850 \mathrm{mg} / \mathrm{m}^{2}$ BID was considered since all of our patients had progression on full dose fluorouracil and or capecitabine chemotherapy and a lower dose seemed feasible to administer as maintenance as well as to preserve quality of life.

Angiogenesis is required for tumor growth and metastases of colorectal cancer. Even though antiangiogenic drugs have had a significant impact on treatment outcomes in patients with colorectal cancers, eventually all patients in the metastatic setting develop disease progression, presumably due to tumor resistance to anti-angiogenic therapy and chemotherapy (25). Two modes of resistance to anti-angiogenic therapy have been proposed. Intrinsic resistance is defined by the absence of any beneficial effect of anti-angiogenic therapy, even a transitory one leading to no tumor response to therapy. Whereas, adaptive (evasive) resistance refers to the ability of the tumor, after an initial response phase, to develop an adaptation to circumvent specific angiogenic blockade. One of the mechanisms of evasive resistance to an antiangiogenic regimen involves upregulation of alternative pro-angiogenic signaling pathways. Blocking one of these alternate pathways may affect a tumor's ability to continue to grow and metastasize $(26,27)$. IL- 8 is a cytokine, derived by monocytes and macrophages. Few studies have implied the role of IL-8 in angiogenesis as well as in causing resistance to anti-angiogenic chemotherapy agents. A study by Koch et al. demonstrated that human recombinant IL-8 showed angiogenic activity by inducing proliferation and chemotaxis of human umbilical vein endothelial cells. The study also showed that, antibodies to IL- 8 blocked the angiogenic activity in human rheumatoid synovial tissue macrophages, demonstrating the role of IL-8 in angiogenesis (28). Another study by Huang et al. has shown that IL-8 may be responsible for causing resistance to antiangiogenic chemotherapy agents. Sunitinib, is an antiangiogenic targeted agent approved for the treatment of clear cell renal cell carcinoma (RCC). But, patients develop resistance to sunitinib within a year causing further progressive disease (29). This study by Huang et al. developed xenograft models representing sunitinib resistance in RCC. Sunitinib resistant tumors showed increased secretion of IL- 8 and administration of IL-8 antibody re-sensitized the tumors to sunitinib treatment, demonstrating the role of IL-8 not only in angiogenesis but also causing resistance to anti-angiogenic agents (30). IL-8 has been shown to be associated with proliferation, migration, angiogenesis and chemo-sensitivity in vitro and in vivo in colon cancer cell lines models (31). IL-8 and its receptor CXCR2 are demonstrated to be upregulated in colorectal cancer and has been shown to impact in vivo angiogenesis as well as migration, invasion and proliferation of colorectal cancer cells and hence are an excellent therapeutic target (32).

Levocetirizine is a third-generation, potent, non-sedating anti-histamine $(33,34)$. Levocetirizine has extensive welldocumented anti-inflammatory properties, inhibiting IL-8, and $\mathrm{NF}-\mathrm{\kappa B}$ in vivo (35). In vitro, levocetirizine inhibits the secretion of IL-6 and IL-8 and inhibits the upregulation of $\mathrm{NF}-\kappa \mathrm{B}(36)$. 
We conducted this trial to treat patients with refractory colorectal cancer with a combination regimen including capecitabine plus bevacizumab, an anti-angiogenic agent and added an IL-8 inhibitor, levocetirizine, to study the effects on patients' tumor response and the systemic cytokine profile. The primary endpoint of the trial was to determine median progression-free survival in patients treated with this combination. The secondary endpoint included evaluation of tolerability of this regimen and an exploratory endpoint was to evaluate the relationship between the cytokine profile and response or resistance to anti-angiogenic therapy.

\section{Methods}

\section{Patients and study design}

This was a randomized single institutional phase II trial. Eligibility criteria included histologically or cytologically confirmed diagnosis of CRC, measurable progressive disease refractory to prior therapy with fluoropyrimidine, oxaliplatin, irinotecan, and/or anti-angiogenic therapy and epidermal growth factor receptor (EGFR) inhibitor such as cetuximab or panitumumab for KRAS wild type tumors. Other inclusion criteria included adequate bone marrow and organ function and ECOG of $<2$. Patients with active brain metastasis, dihydropyrimidine dehydrogenase (DPD) deficiency, uncontrolled hypertension, proteinuria, unstable angina and known HIV status on highly active antiretroviral therapy (HAART) were excluded from the trial due to possible interaction of levocetirizine with HAART medications. The study protocol, NCT01722162 and ethics approval was obtained from Washing University Institutional Review Board (IRB) and was allotted with Human Research Protection Office (HRPO) number \#201303043 and all the participants gave informed consent before participating in the trial.

\section{Treatment plan}

Patients were randomized to one of two arms: patients in Arm A initiated the therapy with bevacizumab + capecitabine and started taking levocetirizine daily on Day 8 of Cycle 1. Patients in Arm B initiated levocetirizine daily 7 days prior to initiation of therapy with bevacizumab + capecitabine (Day-7).

\section{Drug administration}

Bevacizumab was given IV on an outpatient basis at a dose of $5 \mathrm{mg} / \mathrm{kg}$ on Day 1 of each 2-week cycle. Capecitabine was given orally at a dose of $850 \mathrm{mg} / \mathrm{m}^{2}$ twice a day on Days 1-7 of each 2-week cycle. Levocetirizine was also given orally at a dose of $5 \mathrm{mg}$ daily before bed. Patients were followed every 2 weeks up to 30 days after progression of disease, or until death, whichever occurred first.

\section{Assessment}

Patients received CT scans at baseline and at every 8 weeks. Response and progression were evaluated by using Response Evaluation Criteria in Solid Tumors (RECIST) guideline version 1.1. All adverse events and toxicity grading report was done according to the revised NCI Common Terminology Criteria for Adverse Events (CTCAE) version 4.0.

\section{Statistical consideration}

The study randomization (Arm A and Arm B) was to assess the systemic cytokine response. The primary end point was progression free survival (PFS) and secondary endpoints included ORR and tolerability. Since all patients received the same overall therapy, both arms were combined in evaluating the incidence and severity of adverse events.

\section{Expected effect size, sample size and study power}

Phase III randomized double-blind, placebo-controlled multicenter trial (CORRECT) (21), results showed that the median PFS in patients with metastatic colorectal cancer who had progression during or less than three months after their last standard therapy was 1.7 months. Based on this historical control, we proposed a favorable PFS of 3.4 months for our study regimen (IL-8 inhibitor plus capecitabine and bevacizumab). With a sample size of 36 evaluable patients (Arms A and B combined) the study had the power $\geq 0.90$ to identify a doubling of median PFS (1.7 to 3.4 months) at a 0.05 significance level. The proposed sample size also had the power $\geq 0.80$ to identify a difference between median PFS of 1.7 and 3.0 months at the same significance level. The sample size of 36 evaluable patients seemed adequate for the primary aim and included a margin for error such that the minimal detectable difference with 36 patients is an increase of $75 \%$ in median PFS. 


\section{Analysis plan}

Cox proportional hazards models were used to estimate median PFS with a $95 \%$ confidence interval. Binomial proportions and $95 \%$ confidence intervals were used to describe the incidence of adverse events. Chi-square goodness-of-fit tests was used to test for increased severity (grade) of adverse events, especially of grades 3 and 4 over 1 and 2. Tables and multidimensional plots were used to illustrate cytokine expression by study arm, and multiplicity corrected t-tests or Wilcoxon rank sum tests were used to test for differences between study arms.

\section{Correlative studies}

Cytokine measurement: collection of specimen(s)

A total of $6 \mathrm{~mL}$ of blood was collected in 2 EDTA tubes in Arm A prior to initiation of capecitabine and bevacizumab (baseline or Cycle 1 Day 1) and prior to initiation of levocetirizine (Cycle 1 Day 8) and in Arm B prior to initiation of levocetirizine (baseline or Day-7) and prior to initiation of capecitabine and bevacizumab (Cycle 1 Day 1). Blood for cytokine measurement was also collected in both the arms on cycle 2 Day 1, prior to infusion of bevacizumab and on cycle 3 Day 1, prior to infusion of bevacizumab. Cytokines were measured with ELISA method from R\&D Systems as per protocol by Ciprandi et al. (37).

\section{Results}

\section{Study population}

Forty-seven patients were consented for the trial from 4/26/2013 until 7/15/2015. Five patients in Arm A and six patients in Arm B were not enrolled (NE) due to either insurance denial, disease progression prior to starting the trial or withdrawal of consent or a trial unrelated adverse event. Thirty-six evaluable patients were randomly assigned to Arm A and Arm B and treated per trial protocol. Table 1 demonstrates the baseline characteristics of all 47 patients in Arm A and B. Median age on the trial was 60 years. Majority of the patients had a history of colon cancer with prior history of surgical resection. All the patients on the trial had received at least two or more lines of systemic chemotherapy prior to their enrollment. Nineteen percent of patients had history of rectal cancer and had received neoadjuvant chemoradiation and surgical resection as a part of their initial treatment. KRAS was mutated in 24 out of 47 patients. KRAS status was unknown in one patient. Eighty percent of patients had more than one site of metastatic disease. Forty-five out of 47 patients had prior bevacizumab exposure and all 36 patients included in the efficacy analysis had prior bevacizumab therapy.

\section{Efficacy}

All 36 patients on the trial had either progression of their disease or had died, hence, there were no censored (alive at last follow up) observations in the model of progression-free survival. Table 2 demonstrates efficacy and PFS in arm A and arm B. Median PFS was 3.36 months in Arm A and 3.5 months in Arm B. Twenty patients out of 36 (55\%) showed stable disease. Figure 1 shows a Kaplan Meier survival curve demonstrating PFS in both Arm and Arm B. The curves overlap indicating similar PFS in both arms of the study.

\section{Toxicity}

Table 3 describes all adverse events including grade 3 and 4 adverse events related to the treatment. Treatment-related death did not occur in any of the arms in the trial. The majority of the patients tolerated this regimen very well. The most common side effects were grade 1 constipation, nausea, diarrhea, vomiting, abdominal pain, fatigue, headaches and cough as shown in Table 3. There were no grade 4 adverse events in Arm A but 1 patient in Arm B suffered respiratory failure which was considered grade 4 adverse event related to progressive disease. The adverse event profile of the combination study regimen was within the expected margins of this regimen. There were no new, unanticipated toxicities noted.

\section{Correlative studies}

Collection of cytokine IL-8 levels were attempted on all 36 evaluable patients. Blood samples of four patients in Arm A and five patients in Arm B were considered non-measurable due to either missing samples or due to processing delays of the blood samples. Figure 2 and Figure 3 show the IL-8 levels across Arm A and Arm B respectively. One patient in Arm B had cytokine IL-8 levels four-fold higher than mean IL-8 levels across Arm B. Figure 4 shows the best response by IL-8 levels in both arms combined. In general IL-8 levels were greater in patients with progressive disease than those with stable disease. 
Table 1 Patient baseline characteristics $(\mathrm{N}=47)$

\begin{tabular}{|c|c|c|c|c|}
\hline Characteristics & $\mathrm{N}$ & $\%$ & Arm A & Arm B \\
\hline Male & 29 & 61.7 & 14 & 15 \\
\hline Female & 18 & 38.3 & 9 & 9 \\
\hline Median age & 60 & & 62 & 58 \\
\hline Colon & 30 & 63.8 & 15 & 15 \\
\hline Rectum & 9 & 19.1 & 3 & 6 \\
\hline Rectosigmoid & 8 & 17.0 & 5 & 3 \\
\hline \multicolumn{5}{|l|}{ Prior therapy } \\
\hline Radiotherapy & 9 & 19.1 & 3 & 6 \\
\hline Bevacizumab & 45 & 95.8 & 23 & 22 \\
\hline \multicolumn{5}{|l|}{ K-ras status } \\
\hline Mutated & 24 & 51.1 & 14 & 10 \\
\hline Wild type & 22 & 46.8 & 8 & 14 \\
\hline Unknown & 1 & 2.1 & 1 & - \\
\hline \multicolumn{5}{|l|}{ Metastatic site } \\
\hline Lung only & 5 & 10.6 & 1 & 4 \\
\hline
\end{tabular}

LN, lymph nodes.

Table 2 Efficacy $(\mathrm{N}=47)$

\begin{tabular}{lccccc}
\hline Study Arm & NE patients N & PD patients N [\%] & SD patients N [\%] & Total patients (N) & $\begin{array}{c}\text { Median PFS in } \\
\text { months }\end{array}$ \\
\hline Arm A & 5 & 8 & 10 & 23 & 3.36 \\
Arm B & 6 & 8 & 10 & 24 & 3.50 \\
Total patients & 11 & $16[44]$ & $20[55]$ & 47 & - \\
\hline
\end{tabular}

NE, not evaluable; PD, progressive disease; SD, stable disease; PFS, progression free survival.

\section{Discussion}

Colorectal cancers are vascular tumors which rely on VEGF mediated angiogenesis for tumor growth and progression. VEGF expression by CRCs is associated with increased metastasis and poor prognosis $(38,39)$. Therapeutic blockade of VEGF signalling has been successfully deployed as a treatment strategy to treat metastatic CRC (mCRC) and there are FDA approved drugs for the treatment of these cancers that affect the angiogenesis pathway. Disappointingly, however, all patients eventually develop resistance to antiangiogenic therapy and succumb to their cancer. Bevacizumab, 


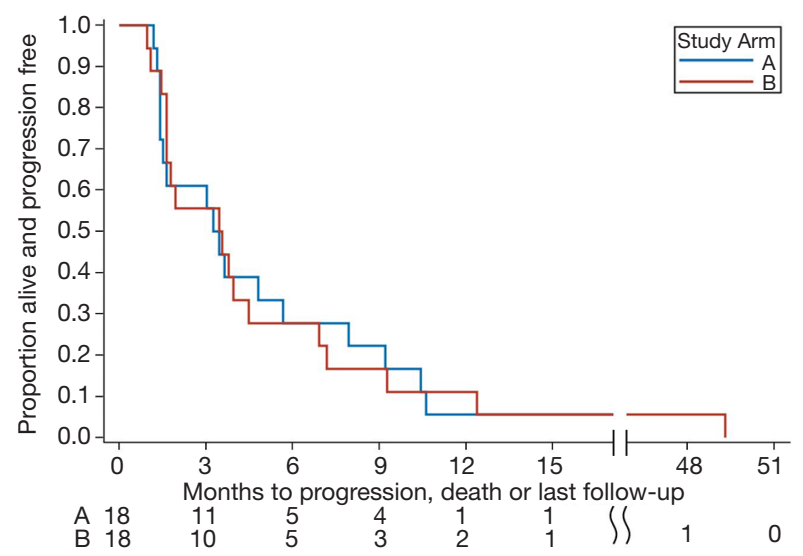

Figure 1 Progression free survival: summary of deaths and progression events. a humanized monoclonal antibody against VEGF, prevents interaction between VEGF and VEGFR-2, acts as an antiangiogenic agent and is approved along with chemotherapy as first and second line treatment for mCRC and contributes to an improvement in overall survival. Capecitabine with bevacizumab is an established and effective maintenance regimen in metastatic colorectal cancer patients after induction chemotherapy per phase III CAIRO 3 study (40). Our study showed that, this regimen was also well tolerated in refractory metastatic colorectal cancer patients and did not affect their quality of life. None of the patients in our study discontinued treatment due to regimen toxicity.

A number of mechanisms of resistance (26) to antiangiogenic therapies have been described, including the increased expression of various cytokines (IL-8, IL-6,

Table 3 Adverse events related to the treatment including grade 3 and grade 4 adverse events

\begin{tabular}{|c|c|c|c|c|}
\hline Adverse events & Grade 1, n (\%) & Grade 2, n (\%) & Grade 3, n (\%) & Grade 4, n (\%) \\
\hline Heart failure & & & $1(2.78)$ & \\
\hline Colon fistula/perforation & & & $2(5.56)$ & \\
\hline Fatigue & $15(41.67)$ & $6(16.67)$ & $2(5.56)$ & \\
\hline Bacteremia & & & $2(5.56)$ & \\
\hline Anorexia & 12 (33.34) & & $1(2.78)$ & \\
\hline UTI & & & $1(2.78)$ & \\
\hline Urinary retention & & & $1(2.78)$ & \\
\hline $\begin{array}{l}\text { Generalized muscle } \\
\text { weakness }\end{array}$ & & & $2(5.56)$ & \\
\hline Respiratory failure & & & & $1(2.77)$ \\
\hline Hypertension & & & $3(8.34)$ & \\
\hline Thromboembolic event & & & $1(2.78)$ & \\
\hline Abdominal pain & $6(16.67)$ & & & \\
\hline Constipation & $11(30.56)$ & & & \\
\hline Diarrhea & $14(38.89)$ & $3(8.34)$ & & \\
\hline Nausea & $14(38.89)$ & & & \\
\hline Vomiting & $10(27.78)$ & $2(5.56)$ & & \\
\hline
\end{tabular}

LFT, liver function tests; UTI, urinary tract infection. 


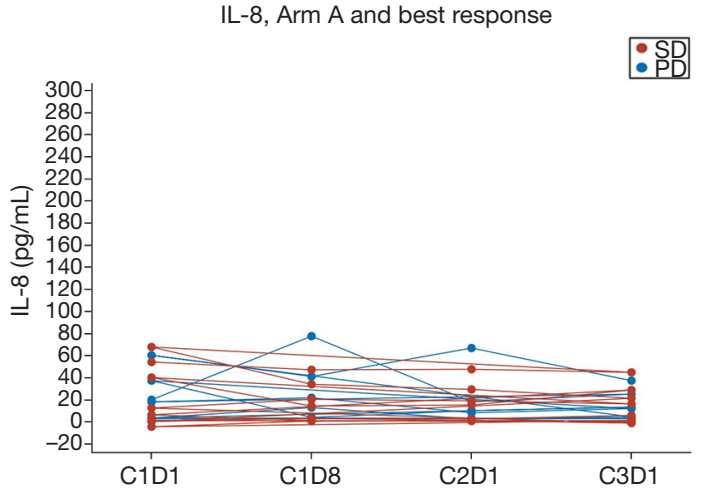

Figure 2 IL-8 levels across Arm A. SD, stable disease; PD, progressive disease.

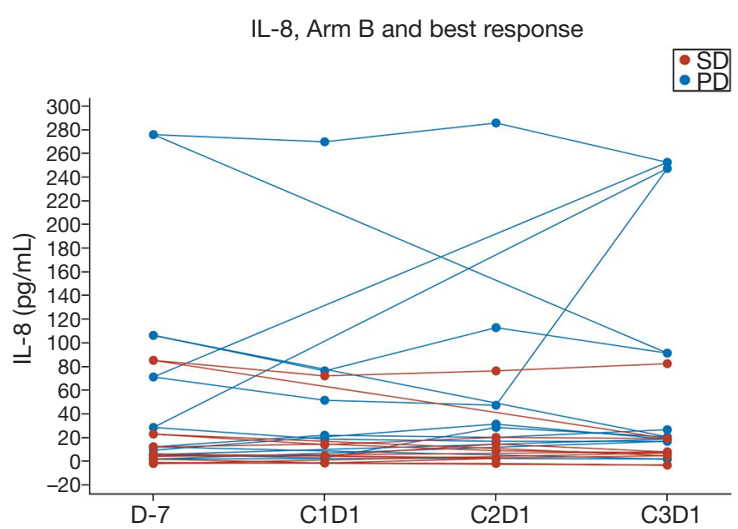

Figure 3 IL-8 levels across Arm B. SD, stable disease; PD, progressive disease.

IL-17, etc.) $(31,41,42)$ and increased activity of immunemodulatory cells from the tumor microenvironment but there are no known biomarkers to predict sensitivity or resistance to anti-angiogenic therapies. We selected levocetirizine, a potent anti-histamine, is commercially available and has shown inhibition of IL- 8 and NF-kB in in vivo and in vitro studies $(36,43,44)$.

In our study, fifty-five percent of patients on our study had stable disease as best response. This was despite $100 \%$ of patients with prior bevacizumab exposure. The PFS was 3.5 months and was similar in both the arms of the study. This PFS was better than other regimens used in the refractory setting such as regorafenib and trifluridine/ tipiracil which showed median progression free survival of 3.2 and 2.0 months respectively $(17,18)$.

Evaluation of cytokine IL-8 levels in our study did not show

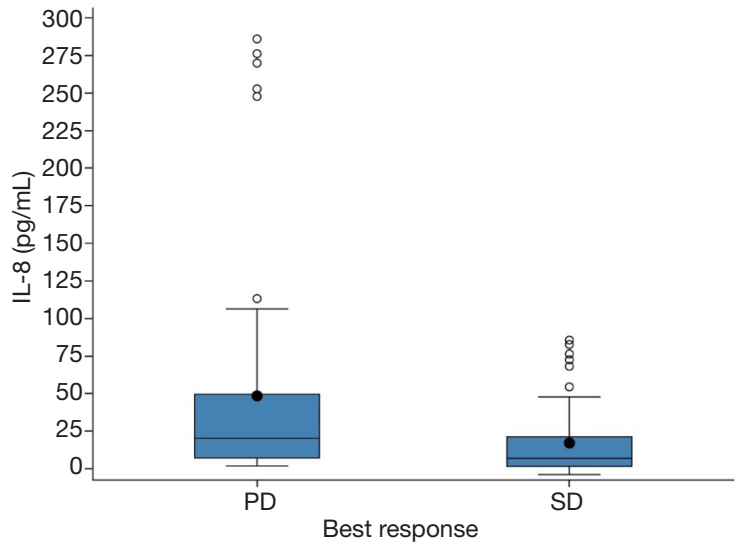

Figure 4 IL-8 levels by best response in both arms combined.

any correlation with disease progression. In both arms, patients with stable disease had lower levels of IL-8 at baseline and throughout first three cycles of chemotherapy as compared to patients with progressive disease. We used an ELISA method to calculate circulating IL-8 levels, however, ELISA based assays have their own limitations in terms of the quality of antibody used as well as narrow dynamic range which may have falsely reduced the concentration of circulating cytokine in the blood due to a dilution effect (45). Also, ELISA results may have been less precise due to our small sample size.

In conclusion, the regimen of levocetirizine with capecitabine and bevacizumab was well tolerated by refractory mCRC patients without any added toxicity from levocetirizine. The combination of levocetirizine with capecitabine and bevacizumab showed comparable PFS estimates to other commonly used regimens. We were unable to demonstrate an ability to overcome resistance to anti-angiogenic therapy using a commercially feasible cytokine inhibition strategy, a good avenue of investigative therapeutic strategy to overcome angiogenic resistance.

\section{Acknowledgements}

None.

\section{Footnote}

Conflicts of Interest: The authors have no conflicts of interest to declare.

Etbical Statement: The ethics approval was obtained from Washing University Institutional Review Board (IRB) 
and was allotted with Human Research Protection Office (HRPO) number \#201303043 and all the participants gave informed consent before participating in the trial.

\section{References}

1. Siegel RL, Miller KD, Jemal A. Cancer statistics, 2018. CA Cancer J Clin 2018;68:7-30.

2. Van Cutsem E, Oliveira J. Advanced colorectal cancer: ESMO clinical recommendations for diagnosis, treatment and follow-up. Ann Oncol 2009;20 Suppl 4:61-3.

3. Giantonio BJ, Catalano PJ, Meropol NJ, et al. Bevacizumab in combination with oxaliplatin, fluorouracil, and leucovorin (FOLFOX4) for previously treated metastatic colorectal cancer: results from the Eastern Cooperative Oncology Group Study E3200. J Clin Oncol 2007;25:1539-44.

4. Hurwitz H, Fehrenbacher L, Novotny W, et al. Bevacizumab plus irinotecan, fluorouracil, and leucovorin for metastatic colorectal cancer. $\mathrm{N}$ Engl J Med 2004;350:2335-42.

5. Kabbinavar FF, Hambleton J, Mass RD, et al. Combined analysis of efficacy: the addition of bevacizumab to fluorouracil/leucovorin improves survival for patients with metastatic colorectal cancer. J Clin Oncol 2005;23:3706-12.

6. Kubicka S, Greil R, Andre T, et al. Bevacizumab plus chemotherapy continued beyond first progression in patients with metastatic colorectal cancer previously treated with bevacizumab plus chemotherapy: ML18147 study KRAS subgroup findings. Ann Oncol 2013;24:2342-9.

7. Amado RG, Wolf M, Peeters M, et al. Wild-type KRAS is required for panitumumab efficacy in patients with metastatic colorectal cancer. J Clin Oncol 2008;26:1626-34.

8. Andre T, Louvet C, Maindrault-Goebel F, et al. CPT-11 (irinotecan) addition to bimonthly, high-dose leucovorin and bolus and continuous-infusion 5 -fluorouracil (FOLFIRI) for pretreated metastatic colorectal cancer. GERCOR. Eur J Cancer 1999;35:1343-7.

9. Colucci G, Gebbia V, Paoletti G, et al. Phase III randomized trial of FOLFIRI versus FOLFOX4 in the treatment of advanced colorectal cancer: a multicenter study of the Gruppo Oncologico Dell'Italia Meridionale. J Clin Oncol 2005;23:4866-75.

10. Falcone A, Ricci S, Brunetti I, et al. Phase III trial of infusional fluorouracil, leucovorin, oxaliplatin, and irinotecan (FOLFOXIRI) compared with infusional fluorouracil, leucovorin, and irinotecan (FOLFIRI) as firstline treatment for metastatic colorectal cancer: the Gruppo Oncologico Nord Ovest. J Clin Oncol 2007;25:1670-6.

11. Kohne CH, Hofheinz R, Mineur L, et al. First-line panitumumab plus irinotecan/5-fluorouracil/leucovorin treatment in patients with metastatic colorectal cancer. J Cancer Res Clin Oncol 2012;138:65-72.

12. Saltz LB, Clarke S, Diaz-Rubio E, et al. Bevacizumab in combination with oxaliplatin-based chemotherapy as firstline therapy in metastatic colorectal cancer: a randomized phase III study. J Clin Oncol 2008;26:2013-9.

13. Tabernero J, Yoshino T, Cohn AL, et al. Ramucirumab versus placebo in combination with second-line FOLFIRI in patients with metastatic colorectal carcinoma that progressed during or after first-line therapy with bevacizumab, oxaliplatin, and a fluoropyrimidine (RAISE): a randomised, double-blind, multicentre, phase 3 study. Lancet Oncol 2015;16:499-508.

14. Van Cutsem E, Kohne CH, Hitre E, et al. Cetuximab and chemotherapy as initial treatment for metastatic colorectal cancer. N Engl J Med 2009;360:1408-17.

15. Li J, Qin S, Xu R, et al. Regorafenib plus best supportive care versus placebo plus best supportive care in Asian patients with previously treated metastatic colorectal cancer (CONCUR): a randomised, double-blind, placebocontrolled, phase 3 trial. Lancet Oncol 2015;16:619-29.

16. Van Cutsem E, Tabernero J, Lakomy R, et al. Addition of aflibercept to fluorouracil, leucovorin, and irinotecan improves survival in a phase III randomized trial in patients with metastatic colorectal cancer previously treated with an oxaliplatin-based regimen. J Clin Oncol 2012;30:3499-506.

17. Yoshino T, Komatsu Y, Yamada Y, et al. Randomized phase III trial of regorafenib in metastatic colorectal cancer: analysis of the CORRECT Japanese and non-Japanese subpopulations. Invest New Drugs 2015;33:740-50.

18. Yoshino T, Mizunuma N, Yamazaki K, et al. TAS-102 monotherapy for pretreated metastatic colorectal cancer: a double-blind, randomised, placebo-controlled phase 2 trial. Lancet Oncol 2012;13:993-1001.

19. Le DT, Uram JN, Wang H, et al. PD-1 Blockade in Tumors with Mismatch-Repair Deficiency. N Engl J Med 2015;372:2509-20.

20. Topalian SL, Hodi FS, Brahmer JR, et al. Safety, activity, and immune correlates of anti-PD-1 antibody in cancer. $\mathrm{N}$ Engl J Med 2012;366:2443-54.

21. Grothey A, Van Cutsem E, Sobrero A, et al. Regorafenib 
monotherapy for previously treated metastatic colorectal cancer (CORRECT): an international, multicentre, randomised, placebo-controlled, phase 3 trial. Lancet 2013;381:303-12.

22. Heinemann V, von Weikersthal LF, Decker T, et al. FOLFIRI plus cetuximab versus FOLFIRI plus bevacizumab as first-line treatment for patients with metastatic colorectal cancer (FIRE-3): a randomised, open-label, phase 3 trial. Lancet Oncol 2014;15:1065-75.

23. Tournigand C, Andre T, Achille E, et al. FOLFIRI followed by FOLFOX6 or the reverse sequence in advanced colorectal cancer: a randomized GERCOR study. J Clin Oncol 2004;22:229-37.

24. Scheithauer W, Kornek GV, Raderer M, et al. Randomized multicenter phase II trial of two different schedules of capecitabine plus oxaliplatin as first-line treatment in advanced colorectal cancer. J Clin Oncol 2003;21:1307-12.

25. Ebos JM, Lee CR, Kerbel RS. Tumor and host-mediated pathways of resistance and disease progression in response to antiangiogenic therapy. Clin Cancer Res 2009; 15:5020-5.

26. Bergers G, Hanahan D. Modes of resistance to antiangiogenic therapy. Nat Rev Cancer 2008;8:592-603.

27. Giuliano S, Pages G. Mechanisms of resistance to antiangiogenesis therapies. Biochimie 2013;95:1110-9.

28. Koch AE, Polverini PJ, Kunkel SL, et al. Interleukin-8 as a macrophage-derived mediator of angiogenesis. Science 1992;258:1798-801.

29. Motzer RJ, Hutson TE, Tomczak P, et al. Sunitinib versus interferon alfa in metastatic renal-cell carcinoma. $\mathrm{N}$ Engl J Med 2007;356:115-24.

30. Huang D, Ding Y, Zhou M, et al. Interleukin-8 mediates resistance to antiangiogenic agent sunitinib in renal cell carcinoma. Cancer Res 2010;70:1063-71.

31. Ning Y, Manegold PC, Hong YK, et al. Interleukin-8 is associated with proliferation, migration, angiogenesis and chemosensitivity in vitro and in vivo in colon cancer cell line models. Int J Cancer 2011;128:2038-49.

32. Ning Y, Lenz HJ. Targeting IL-8 in colorectal cancer. Expert Opin Ther Targets 2012;16:491-7.

33. Popov TA, Dumitrascu D, Bachvarova A, et al. A comparison of levocetirizine and desloratadine in the histamine-induced wheal and flare response in human skin in vivo. Inflamm Res 2006;55:241-4.

34. Shih MY, Hsu JY, Weng YS, et al. Influence of cetirizine and levocetirizine on two cytokines secretion in human airway epithelial cells. Allergy Asthma Proc 2008;29:480-5.
35. Ciprandi G, Cirillo I, Vizzaccaro A, et al. Levocetirizine improves nasal obstruction and modulates cytokine pattern in patients with seasonal allergic rhinitis: a pilot study. Clin Exp Allergy 2004;34:958-64.

36. Jang YJ, Wang JH, Kim JS, et al. Levocetirizine inhibits rhinovirus-induced ICAM-1 and cytokine expression and viral replication in airway epithelial cells. Antiviral Res 2009;81:226-33.

37. RnD systems quantikine IL8 manual. Available online: https://resources.rndsystems.com/pdfs/datasheets/ d8000c.pdf

38. Ellis LM, Takahashi Y, Liu W, et al. Vascular endothelial growth factor in human colon cancer: biology and therapeutic implications. Oncologist 2000;5 Suppl 1:11-5.

39. Grothey A, Allegra C. Antiangiogenesis therapy in the treatment of metastatic colorectal cancer. Ther Adv Med Oncol 2012;4:301-19.

40. Simkens LH, van Tinteren H, May A, et al. Maintenance treatment with capecitabine and bevacizumab in metastatic colorectal cancer (CAIRO3): a phase 3 randomised controlled trial of the Dutch Colorectal Cancer Group. Lancet 2015;385:1843-52.

41. Chung AS, Wu X, Zhuang G, et al. An interleukin-17mediated paracrine network promotes tumor resistance to anti-angiogenic therapy. Nat Med 2013;19:1114-23.

42. Malicki S, Winiarski M, Matlok M, et al. IL-6 and IL-8 responses of colorectal cancer in vivo and in vitro cancer cells subjected to simvastatin. J Physiol Pharmacol 2009;60:141-6.

43. Ciprandi G, Cirillo I, Vizzaccaro A, et al. Desloratadine and levocetirizine improve nasal symptoms, airflow, and allergic inflammation in patients with perennial allergic rhinitis: a pilot study. Int Immunopharmacol 2005;5:1800-8.

44. Ciprandi G, Cirillo IG, Vizzaccaro A, et al. Levocetirizine improves nasal symptoms and airflow in patients with persistent allergic rhinitis: a pilot study. Eur Ann Allergy Clin Immunol 2005;37:25-9.

45. Aziz N, Nishanian P, Mitsuyasu R, et al. Variables that affect assays for plasma cytokines and soluble activation markers. Clin Diagn Lab Immunol 1999;6:89-95.

Cite this article as: Amin M, Desai $M$, Trinkaus K, Brown A, Wang-Gillam A, Tan B, Picus J, Sorscher S, Highkin M, Lears K, Lockhart AC. Phase II trial of levocetirizine with capecitabine and bevacizumab to overcome the resistance of antiangiogenic therapies in refractory metastatic colorectal cancer. J Gastrointest Oncol 2019;10(3):412-420. doi: 10.21037/jgo.2019.02.01 